\title{
Enzymatic production of 4-O-methyl D-glucaric acid from hardwood xylan
}

\author{
Thu V. Vuong ${ }^{1}$ and Emma R. Master ${ }^{1,2^{*}}$
}

\begin{abstract}
Background: Dicarboxylic acids offer several applications in detergent builder and biopolymer fields. One of these acids, 4-O-methyl D-glucaric acid, could potentially be produced from glucuronoxylans, which are a comparatively underused fraction of wood and agricultural biorefineries.

Results: Accordingly, an enzymatic pathway was developed that combines AxyAgu115A, a GH115 a-glucuronidase from Amphibacillus xylanus, and GOOX, an AA7 gluco-oligosaccharide oxidase from Sarocladium strictum, to produce this bio-based chemical from glucuronoxylan. AxyAgu115A was able to release almost all 4-O-methyl D-glucuronic acid from glucuronoxylan while a GOOX variant, GOOX-Y300A, could convert 4-O-methyl D-glucuronic acid to the corresponding glucaric acid at a yield of $62 \%$. Both enzymes worked effectively at alkaline conditions that increase xylan solubility. Given the sensitivity of AxyAgu115A to hydrogen peroxide and optimal performance of GOOX-Y300A at substrate concentrations above $20 \mathrm{mM}$, the two-step enzyme pathway was demonstrated as a sequential, one-pot reaction. Additionally, the resulting xylan was easily recovered from the one-pot reaction, and it was enzymatically hydrolysable.

Conclusions: The pathway in this study requires only two enzymes while avoiding a supplementation of costly cofactors. This cell-free approach provides a new strategy to make use of the underutilized hemicellulose stream from wood and agricultural biorefineries.
\end{abstract}

Keywords: 4-O-Methyl D-glucaric acid, GH115, AA7, Xylan, Hemicellulose, Biorefinery

\section{Background}

Glucuronoxylans are a major form of hemicellulose present in wood and agricultural fiber. For example, between 15 and $30 \%$ of hardwood dry mass is acetylated-glucuronoxylan, whereas up to $10 \%$ of coniferous softwood dry mass is arabino-glucuronoxylan, and up to $30 \%$ of cereal dry mass is glucurono-arabinoxylan [1]. In these glucuronoxylans, the 4-O-methyl D-glucuronic acid (MeGlcA) or D-glucuronic acid (GlcA) substituents are linked to the $\beta$-(1 $\rightarrow 4)$-linked xylose backbone through an $\alpha-(1 \rightarrow 2)$ linkage. The ratio of (Me)GlcA to xylose (Xylp) depends on the xylan source, and is mainly reported as being

\footnotetext{
*Correspondence: emma.master@utoronto.ca

${ }^{1}$ Department of Chemical Engineering and Applied Chemistry, University of Toronto, Toronto, ON, Canada
}

Full list of author information is available at the end of the article between 1:10 [2] and 1:15 [3], but also as high as 1:3 [1], in glucuronoxylan from hardwoods. The varied chemistry of xylans has posed a challenge to their broader use, and xylans remain a comparatively underused fraction of wood and agricultural fiber. Enzymatic release of MeGlcA present in glucuronoxylans, however, could be performed as the first step to methyl glucaric acid production while facilitating the recovery of a simplified xylan stream suitable for conversion to xylitol or highvalue products.

Glucaric acid was listed by the US Department of Energy in 2004 as one of the top 12 bio-based chemicals [4]. This dicarboxylic acid could replace phosphoric acid as a builder component in detergents for calcium and magnesium sequestering [5]. It is also a potential building block for a number of biopolymers including new

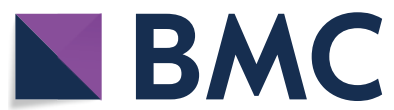

(c) The Author(s) 2020. This article is licensed under a Creative Commons Attribution 4.0 International License, which permits use, sharing, adaptation, distribution and reproduction in any medium or format, as long as you give appropriate credit to the original author(s) and the source, provide a link to the Creative Commons licence, and indicate if changes were made. The images or other third party material in this article are included in the article's Creative Commons licence, unless indicated otherwise in a credit line to the material. If material is not included in the article's Creative Commons licence and your intended use is not permitted by statutory regulation or exceeds the permitted use, you will need to obtain permission directly from the copyright holder. To view a copy of this licence, visit http://creativeco mmons.org/licenses/by/4.0/. The Creative Commons Public Domain Dedication waiver (http://creativecommons.org/publicdomain/ zero/1.0/) applies to the data made available in this article, unless otherwise stated in a credit line to the data. 
nylons and hyperbranched polyesters [6]. Methyl groups of monomers contributed to the molecular architecture and subsequent properties of their derived biopolymers [7]. Therefore, the methylated form of glucaric acid from glucuronoxylan could bring additional functional properties to the chemical, including higher compatibility with surfactants in detergents and hydrophobic biopolymers.

Presently, glucaric acid is commercially synthesized as glucarate by the non-selective nitric acid oxidation of glucose with a yield of ca. 40\% [8]. This conventional approach as well as recent heterogeneous, metal catalyst methods $[9,10]$ suffers from low selectivity, increasing the cost for downstream separation of glucaric acid from other organic acid by-products [11]. The absence of green technologies for glucaric acid production is one of the reasons for its exclusion from the revised list of new top chemical opportunities from biorefineries [12]. Accordingly, considerable investment has been focused on engineering microorganisms such as E. coli [13] and Saccharomyces cerevisiae [14] to transform glucose into glucaric acid. However, even when a co-substrate, myoinositol was added, the maximum yield was only $56 \%$ [14], which remains inefficient for industrial scale production of glucaric acid. Furthermore, fermentation approaches can complicate product recovery, due to the presence of medium components and other metabolites/intermediates. A cell-free approach to produce 4-O-methyl D-glucaric acid (or methyl glucaric acid, for short) from glucuronoxylan was reported [15], where three enzymes including an endo-xylanase (EC 3.2.1.8), $\alpha$-glucuronidase (EC 3.2.1.139), and uronate dehydrogenase (EC 1.1.1.203) were used in a cocktail or co-localized on a scaffold. The xylanase cleaved glucuronoxylan to various xylo-oligosaccharides, of which some contained MeGlcA. The $\alpha$-glucuronidase then removed MeGlcA residues that were attached to the non-reducing end of short xylooligosaccharides. The released MeGlcA was finally converted to methyl glucaric acid by the dehydrogenase [15]. Notably, this approach requires a continuous supply of an exogenous cofactor $\left(\mathrm{NAD}^{+}\right)$and the separation of methyl glucaric acid from other soluble xylo-oligosaccharides. To avoid the need for supplementation with expensive and labile $\mathrm{NAD}^{+}$, other in vitro pathways were proposed that utilize seven enzymes, including uronate dehydrogenase and NADH oxidase, for the production of glucaric acid from sucrose $[16,17]$. However, these multi-enzyme cascades require an initial amount of $\mathrm{NAD}^{+}$and a continuous supply of inorganic phosphate. It was also noted that uronate dehydrogenase used in such pathways can release glucaric acid 1,4-lactone rather than the openring form [18].

A GH115 $\alpha$-glucuronidase from Amphibacillus xylanus (AxyAgu115A), which hydrolyzes the $\alpha-(1 \rightarrow 2)$-linkage connecting MeGlcA to internal and terminal substituted regions of xylans [19], has been previously characterized by our group. This enzyme displays the highest relative activity in alkaline conditions among all other characterized GH115 $\alpha$-glucuronidases with known $\mathrm{pH}$ profiles [19]. Meanwhile, we have also characterized a glucooligosaccharide oxidase from Sarocladium strictum (GOOX) [20], which is a member of Auxiliary Activities family 7 (AA7, http://www.cazy.org). GOOX oxidizes a number of monosaccharides and oligosaccharides, including those derived from hemicelluloses [20]. Seventeen GOOX variants were created [20-22], of which, the catalytic efficiency of GOOX-Y300A on glucose is nearly four times higher than that of the wild-type [20]. Like AxyAgu115A, GOOX works effectively at alkaline conditions with the optimum $\mathrm{pH}$ of 10 [23]. In this study, AxyAgu115A and GOOX-Y300A were combined into a sequential one-pot reaction to produce 4-O-methyl D-glucaric acid from glucuronoxylan, without cofactor supplementation (Fig. 1). In addition to this methyl glucaric acid, a simplified, hydrolysable xylan stream was also recovered.

\section{Results and discussion}

Release of 4-O-methyl D-glucuronic acid by AxyAgu 115A

AxyAgu115A was produced with greater than 95\% purity (Additional file 1: Figure S1). Glucuronoxylan is more soluble in alkaline conditions, allowing 6\% glucuronoxylan to be incubated with AxyAgu115A with proper mixing. AxyAgu115A released only MeGlcA from glucuronoxylan, as analyzed by HPAEC-PAD (Additional file 1: Figure S2). Most of MeGlcA was released by AxyAgu115A during $4 \mathrm{~h}$ of incubation (>98\%), and no additional release of MeGlcA was seen after $16 \mathrm{~h}$. The half-life of AxyAgu115A at $40{ }^{\circ} \mathrm{C}$ was $24 \mathrm{~h}$ [19], thus the enzyme remained active during the 16-h hydrolysis. Consistent with earlier findings [3], no trace of GlcA release (the unmethylated form) was detected, even though the sensitivity of HPAEC-PAD for GlcA detection is approximately three times higher than for MeGlcA (Additional file 1: Figure S3). The release of MeGlcA was also confirmed by nanospray ionization ion-trap mass spectrometry (NSI-MS). A mass scan from 100 to $1000 \mathrm{~m} / \mathrm{z}$ showed that MeGlcA and its dimer are the two major peaks in the spectrum (Additional file 1: Figure S4). The simulated spectrum of MeGlcA was also matched well to the acquired spectrum (Additional file 1: Figure S5).

When the glucuronoxylan loading was greater than $6 \%$, the reaction became viscous, preventing mixing; therefore, the concentration of glucuronoxylan was set at $6 \%$. At this substrate loading, the concentration of MeGlcA released after $4 \mathrm{~h}$ (in $25-\mathrm{mL}$ reactions) was $21.6 \pm 1.2 \mathrm{mM}$, calculated based on the MeGlcA 


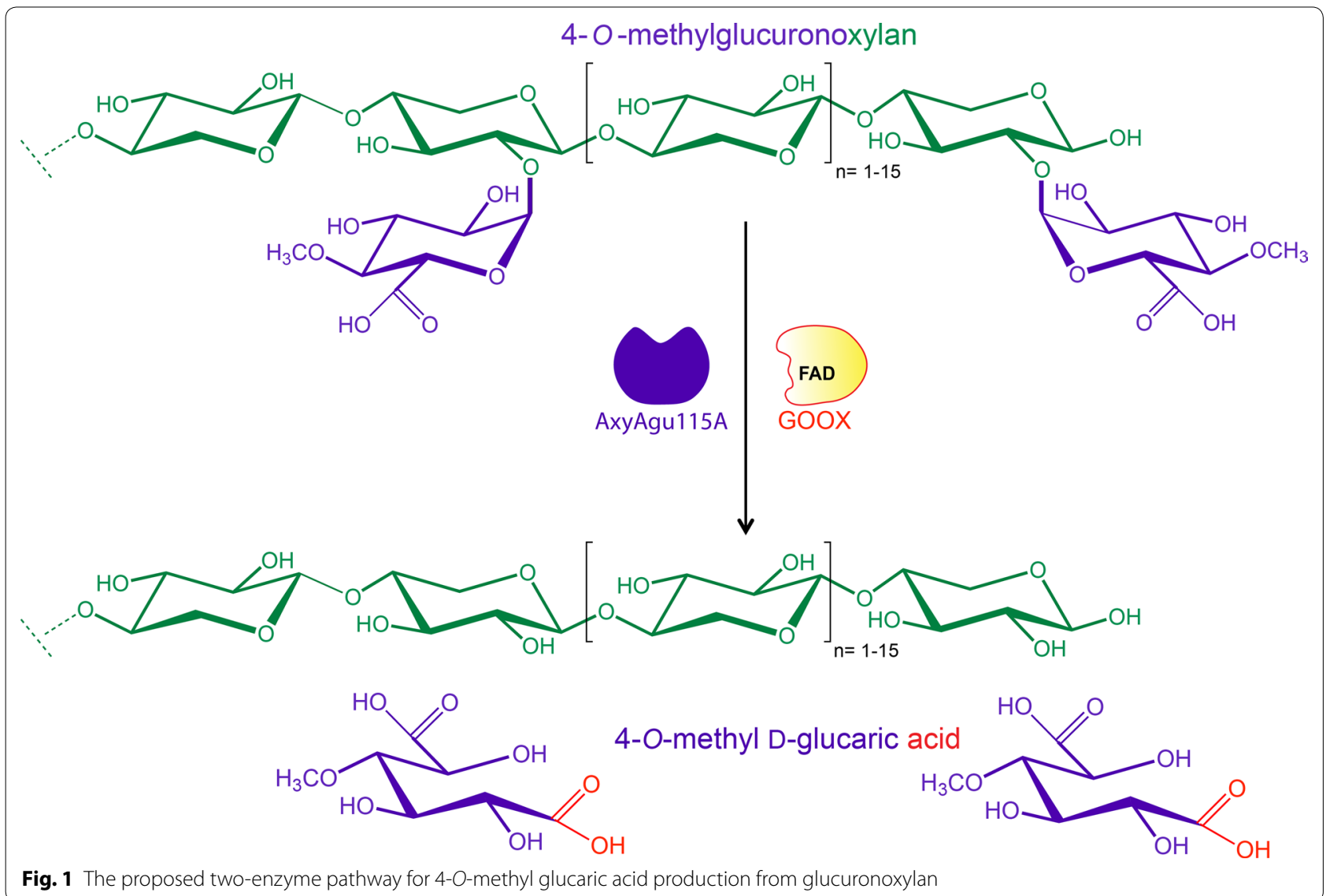

standard curve (Additional file 1: Figure S6). The theoretical maximum molar concentration of MeGlcA, based on a previous analysis of glucuronoxylan composition [3], was $24.4 \mathrm{mM}$; therefore, AxyAgu115A was able to release almost all of MeGlcA present in glucuronoxylan. This finding was supported by methanolysis, where the total concentration of MeGlcA released from glucuronoxylan was measured at approximately $15.6 \mathrm{mM}$. The nearly $20 \%$ lower concentration of MeGlcA released by methanolysis compared to the enzymatic treatment can be explained by partial MeGlcA degradation at high temperature $\left(100{ }^{\circ} \mathrm{C}\right)$ and acid conditions $(2 \mathrm{M} \mathrm{HCl})$ required for methanolysis. A similar percentage of MeGlcA degradation by methanolysis was also previously reported [24].

The $\mathrm{pKa}$ of MeGlcA is 3.0, as predicted by ACD/Labs 2.0 v5 (http://www.ilab.acdlabs.com), so at alkaline conditions, MeGlcA is negatively charged. Therefore, anion-exchange chromatography was used to purify MeGlcA released by AxyAgu115A, which confirmed that the acidic sugar eluted from Dowex resin at ammonium acetate concentrations above $0.5 \mathrm{M}$ (Additional file 1: Figure S7).

\section{Oxidation of 4-0-methyl D-glucuronic acid by GOOX} variants

A preliminary screening of 17 GOOX variants [20, 22] on GlcA and MeGlcA found that the methylated form of D-glucuronic acid was the preferred substrate (Additional file 1: Figure S8); notably, the commercial glucose oxidase did not show any activity on either GlcA or MeGlcA (Additional file 1: Figure S8). One of the GOOX variants, Y300A [20] showed higher specific activity on MeGlcA than other GOOX variants; the Y300A variant also preferred oxidation of MeGlcA over GlcA (Fig. 2). Docking analyses of MeGlcA to the wild-type and Y300A variant showed that the methyl group pointed toward Tyr300, and the replacement of tyrosine with alanine increased hydrogen-bonding to MeGlcA from four to six bonds (Fig. 3). Notably, seven hydrogen bonds are predicted between glucose and the Y300A variant, and performance of the Y300A variant on glucose is higher than that on MeGlcA (see below). This GOOX variant, hereafter GOOX-Y300A, was used for this study (Additional file 1: Figure S1).

The formation of methyl glucaric acid by GOOXY300A was confirmed by NSI-MS (Fig. 4c), where methyl 


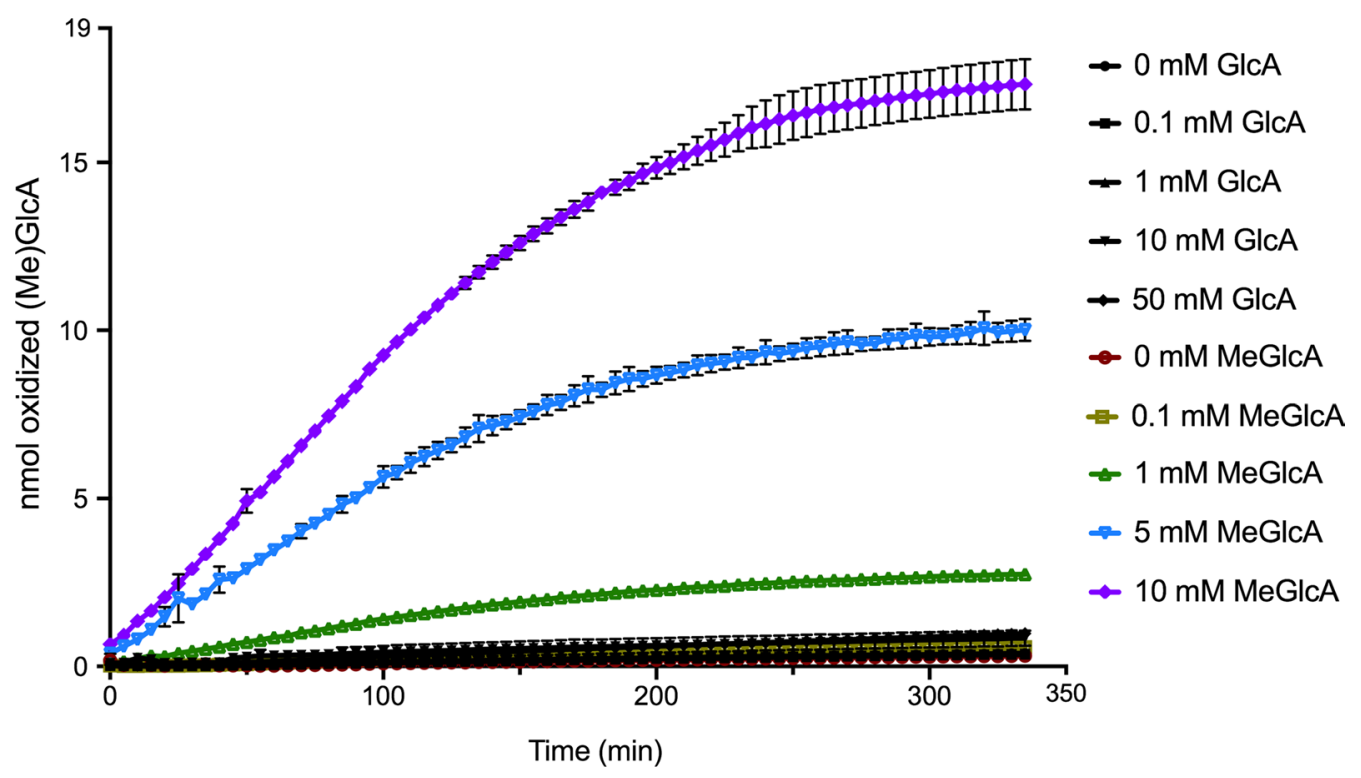

Fig. 2 Substrate preference of GOOX-Y300A. The enzyme ( $16 \mathrm{nM}$ ) was incubated with different concentrations of MeGlcA and GlcA, up to $50 \mathrm{mM}$ in $0.3 \mathrm{M}$ Tris buffer $\mathrm{pH} 8$ at $40^{\circ} \mathrm{C}$, and the amount of oxidized products was determined using a colorimetric assay [23]

glucaric acid was seen at $223.05 \mathrm{~m} / \mathrm{z}$, confirming the oxidation (addition of $15.99 \mathrm{~m} / \mathrm{z}$ ) of MeGlcA $(207.05 \mathrm{~m} / \mathrm{z}$ ). There was a dose response for the production of methyl glucaric acid, as the relative abundance of methyl glucaric acid increased when the substrate concentration was raised from $1 \mathrm{mM}$ to $10 \mathrm{mM}$ (Fig. 4a, b). Consistent with this observation, kinetic analysis of GOOX-Y300A on MeGlcA revealed a $K_{\mathrm{m}}$ of $21 \pm 2 \mathrm{mM}$ and $k_{\text {cat }}$ of $0.91 \pm 0.06 \mathrm{~min}^{-1}$. This $K_{\mathrm{m}}$ value is in the range of MeGlcA concentration that was fully released from $6 \%$ glucuronoxylan, suggesting that GOOX-Y300A should be added to the reaction after the complete action of AxyAgu115A. However, the $K_{\mathrm{m}}$ of GOOX-Y300A on MeGlcA was higher than that on glucose $(8.1 \mathrm{mM})$ while its $k_{\text {cat }}$ was nearly three orders of magnitude lower [20], highlighting the merits of further engineering GOOX-Y300A to increase catalytic performance on MeGlcA.

The conversion yield from MeGlcA to methyl glucaric acid by GOOX-Y300A was 62\%; given methyl glucaric acid was not commercially available, yields were based on MeGlcA consumption quantified by HPAEC-PAD and LC-MS. This yield is $55 \%$ higher than the nitric acid oxidation of glucose to glucaric acid [8]. The conversion yield was not reported by Lee et al. [15], who proposed the in vitro pathway with three enzymes including endo-xylanase, $\alpha$-glucuronidase, and uronate dehydrogenase to produce (methyl)glucaric acid from birchwood xylan. The authors reported a maximum production of $0.7 \mathrm{mM}$ (methyl)glucaric acid from 1\% birchwood xylan after more than $2 \mathrm{~h}$, as quantified by $\mathrm{NADH}$ absorbance at $340 \mathrm{~nm}$ [15]. Provided that a similar xylan source was used, this would be equivalent to a conversion yield of $\sim 20 \%$. Using the same three-enzyme pathway, but with a new thermostable uronate dehydrogenase, Li et al. increased (methyl)glucaric acid yield from beechwood xylan to more than $80 \%$ [25]. Notably, both previously reported methods require a continuous supply of $\mathrm{NAD}^{+}$, motivating $\mathrm{Su}$ et al. to establish a seven-enzyme pathway to incorporate $\mathrm{NAD}^{+}$regeneration [16]. Although the seven-enzyme pathway reached a conversion yield of up $75 \%$ glucaric acid from sucrose in $72 \mathrm{~h}$, the total enzyme loading was more than $2 \mathrm{mg} / \mathrm{mL}$, instead of $0.01 \mathrm{mg} / \mathrm{mL}$ as in two-step pathway reported herein.

Methyl glucaric acid is also chemically produced from MeGlcA using $\mathrm{Ca}(\mathrm{OH})_{2}$ and $\mathrm{NaOH}$; however, the highest yield was only $24 \%$, and the final reaction solution contained eight other dicarboxylic acids [26]. Several approaches that use heterogeneous metal catalysts including $\mathrm{Pt} / \mathrm{C}, \mathrm{Pt} / \mathrm{Au}, \mathrm{Au} / \mathrm{C}$ or $\mathrm{AuBi} / \mathrm{C}$ or $\mathrm{Pt}_{1} \mathrm{Cu}_{3} / \mathrm{TiO}_{2}$ $[9,10]$ could gain a complete conversion of glucose. Unfortunately, the full selectivity of glucose to glucaric acid is not achievable, requiring a separation of glucaric acid from other oxidized products.

\section{Impacts of hydrogen peroxide}

When GOOX-Y300A oxidizes carbohydrates, it utilizes molecular oxygen as a co-substrate, producing hydrogen peroxide. Therefore, the effects of $\mathrm{H}_{2} \mathrm{O}_{2}$ on MeGlcA and enzymatic stability must be considered. No loss of MeGlcA was observed at $100 \mathrm{mM} \mathrm{H}_{2} \mathrm{O}_{2}$ (Additional 


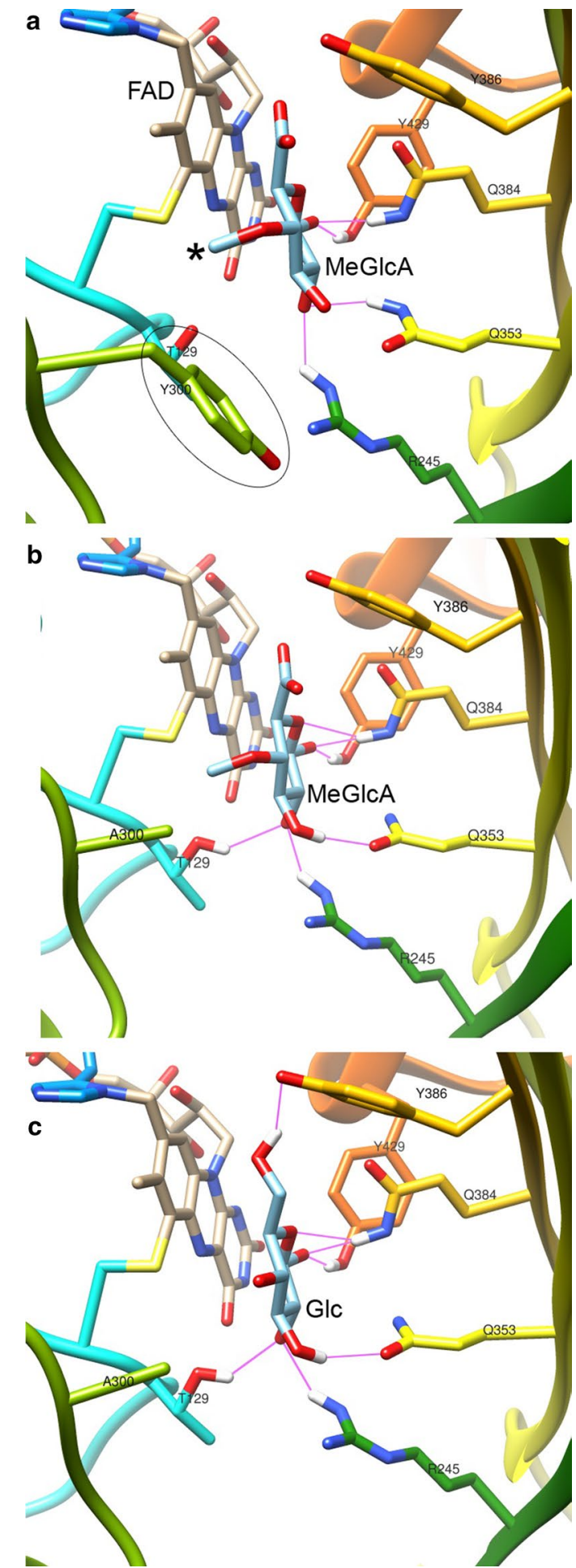

Fig. 3 Docking of MeGlcA and glucose to GOOX variants. a MeGlcA formed four hydrogen bonds (pink line) in the wild-type enzyme, the methyl group (starred) pointed toward the $Y 300$ residue (circled). b The replacement of $\mathrm{Y} 300$ with alanine allowed MeGlcA to form six hydrogen bonds. c Glucose (Glc) formed seven hydrogen bonds with GOOX-Y300A file 1: Figure S9A). Under our conditions, even at the highest glucuronoxylan loading, only about $20 \mathrm{mM}$ $\mathrm{H}_{2} \mathrm{O}_{2}$ could be present as it depends on the released concentration of MeGlcA. Even at $200 \mathrm{mM} \mathrm{H}_{2} \mathrm{O}_{2}$, GOOX-Y300A retains more than $50 \%$ of its activity on glucose and $100 \%$ of its activity on cellobiose [27]. Furthermore, it was reported that $\mathrm{H}_{2} \mathrm{O}_{2}$ is less stable in alkaline conditions, particularly at elevated temperatures $\left(40{ }^{\circ} \mathrm{C}\right)$ [28]. In contrast, AxyAgu115A activity was inactivated when the concentration of $\mathrm{H}_{2} \mathrm{O}_{2}$ was greater than $1 \mathrm{mM}$, and approximately half of AxyAgu115A activity was lost in the presence of $10 \mathrm{mM}$ $\mathrm{H}_{2} \mathrm{O}_{2}$ (Additional file 1: Figure S9B).

\section{Sequential one-pot reaction for methyl glucaric acid production}

Both AxyAgu115A and GOOX-Y300A act at high $\mathrm{pH}$ values and display a similar temperature profile $[19,23]$, confirming the compatibility of these enzymes. Conducting reactions at alkaline conditions offers several advantages, including a higher glucuronoxylan loading $(6 \%$ in this study, compared to $1 \%$ reported in Lee et al. [15]) and a lower presence of lactone forms of glucaric acid [29], which could hinder product recovery. Because the $K_{\mathrm{m}}$ of GOOX-Y300A was at mM levels, and AxyAgu115A was partially inactivated by $\mathrm{H}_{2} \mathrm{O}_{2}$, a onepot sequential reaction was performed where GOOXY300A was added to the reaction after pre-hydrolysis of glucuronoxylan by AxyAgu115A for $4 \mathrm{~h}$. Following $16 \mathrm{~h}$ of incubation with GOOX-Y300A, methyl glucaric acid yields were similar to those achieved using the two-pot sequential system described above (i.e., $60 \%$ yield as confirmed by LC-MS; Fig. 5).

The xylan after AxyAgu115A and GOOX-Y300A treatments formed a hydrogel-like material (Additional file 1: Figure S10), which was separated from the reaction by quick centrifugation $(1 \mathrm{~min}$ at $10,000 \times g)$. This effect on rheological properties was previously correlated to xylan debranching [30]. After washing with MQ water to remove any remaining soluble products, the resulting xylan was still hydrolysable by Novozymes xylanase NS51024 (GH11 xylanase from Thermomyces lanuginosus) (Fig. 6). HPAEC-PAD analyses revealed lower amounts of small oligosaccharides, and less abundance of large/charged oligosaccharide fractions in the resulting xylan (Fig. 6), which is in agreement with its lower reducing-end sugar yield (40\%), compared with the starting glucuronoxylan (Additional file 1: Figure S11). This already washed and neutralized xylan could decrease 

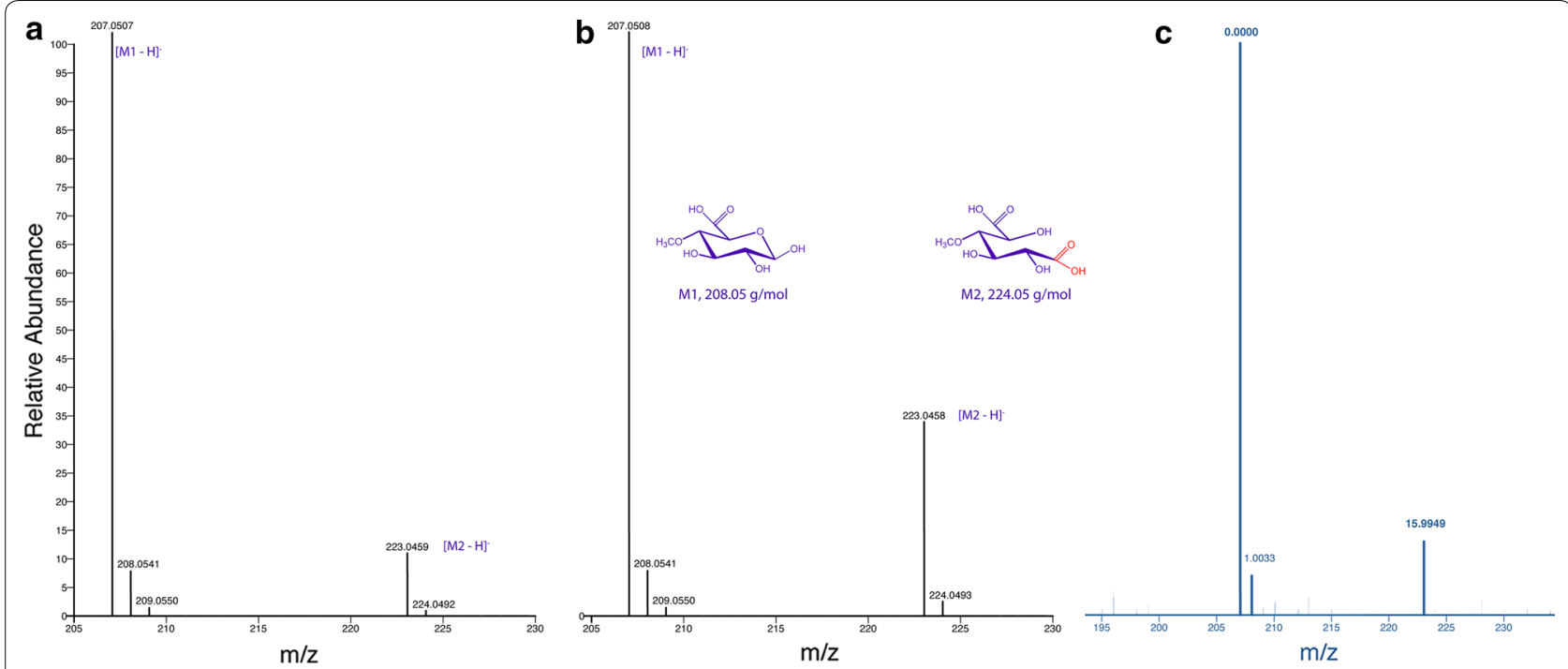

Fig. 4 NSI-MS spectra for the formation of methyl glucaric acid (224.05 g/mol) by GOOX-Y300A. Mass spectra of GOOX-Y300A activity on 1 mM and $10 \mathrm{mM} \mathrm{MeGlcA}$ (a, b, correspondingly); notably, MeGlcA and methyl glucaric acid displayed different ionization potentials. The corrected mass spectrum to confirm the addition of one oxygen $(15.9949 \mathrm{~m} / \mathrm{z})(\mathbf{c})$

downstream separation costs in applications that require high purity of xylan, for instance in xylitol production.

\section{Conclusion}

We report an enzymatic pathway for the production of methyl glucaric acid from glucuronoxylan using two enzymes, AxyAgu115A and GOOX-Y300A. AxyAgu115A released most of MeGlcA directly from glucuronoxylan, as confirmed by methanolysis, whereas GOOX-Y300A converted MeGlcA to methyl glucaric acid at a yield of $62 \%$. Both enzymes worked at alkaline conditions, which permitted reactions using $6 \%$ glucuronoxylan. Because the $K_{\mathrm{m}}$ value of GOOX-Y300A on MeGlcA was $21 \pm 2 \mathrm{mM}$ and AxyAgu115A showed evidence of inhibition above $1 \mathrm{mM} \mathrm{H}_{2} \mathrm{O}_{2}$, these enzymes are suitable for a sequential one-pot reaction where a similar conversion yield was achieved. The xylan co-product can be separated by centrifugation and readily hydrolyzed by a commercial xylanase to xylose and xylo-oligosaccharides, which might be suitable for xylitol or higher value applications.

\section{Materials and methods Materials}

4-O-Methyl glucuronoxylan from beechwood, also known as glucuronoxylan (cat. no. M5144), was purchased from Sigma, USA. 4-O-methyl D-glucuronic acid (MeGlcA, purity $>95 \%$, by $1 \mathrm{H}-\mathrm{NMR}$, cat. no. MG244) was purchased from Synthose Inc., Canada while
D-glucuronic acid (GlcA, not methylated, purity $>98 \%$ by GC, cat.no. G5269) was purchased from Sigma, USA. Commercial enzymes used were catalase (cat. no. C40 from Sigma, USA, $\geq 10,000$ units/mg protein), glucose oxidase (cat. no. G2133 from Sigma, USA), and xylanase (cat. no. NS51024 from Novozymes, Denmark).

\section{Protein production}

AxyAgu115A and GOOX-Y300A were produced based on the previous publications $[19,22]$. Briefly, for AxyAgu115A purification, E. coli BL21( $\lambda \mathrm{DE} 3)$ CodonPlus was grown at $37{ }^{\circ} \mathrm{C}$ in Luria-Bertani medium containing $500 \mathrm{mM}$ sorbitol, $2.5 \mathrm{mM}$ glycine betaine, $34 \mu \mathrm{g} / \mathrm{mL}$ chloramphenicol and $100 \mu \mathrm{g} / \mathrm{mL}$ ampicillin. Cells were induced by $0.5 \mathrm{mM}$ IPTG at $15{ }^{\circ} \mathrm{C}$ for $16 \mathrm{~h}$. Cells were then sonicated in a binding buffer $(300 \mathrm{mM} \mathrm{NaCl}, 50 \mathrm{mM}$ HEPES pH 7.0, 5\% glycerol, and $5 \mathrm{mM}$ imidazole). After centrifugation, the supernatant was incubated with $\mathrm{Ni}-$ NTA resin for $2 \mathrm{~h}$ at $4{ }^{\circ} \mathrm{C}$, and the protein was eluted with an elution buffer (300 mM NaCl, $50 \mathrm{mM}$ HEPES pH 7.0, $5 \% \mathrm{v} / \mathrm{v}$ glycerol, and $250 \mathrm{mM}$ imidazole). The protein was further purified using a Bio-Gel P10 column. For GOOX-Y300A purification, the corresponding Pichia pastoris was grown in BMGY medium, and then induced in BMMY medium with $0.5 \%$ methanol added daily. The secreted GOOX-Y300A protein was harvested using $\mathrm{Ni}-\mathrm{NTA}$ resin as described for AxyAgu115A. The concentration and purity of these recombinant proteins were determined by gel densitometry using a bovine serum albumin (Thermo Fisher Scientific, USA) as the standard. 


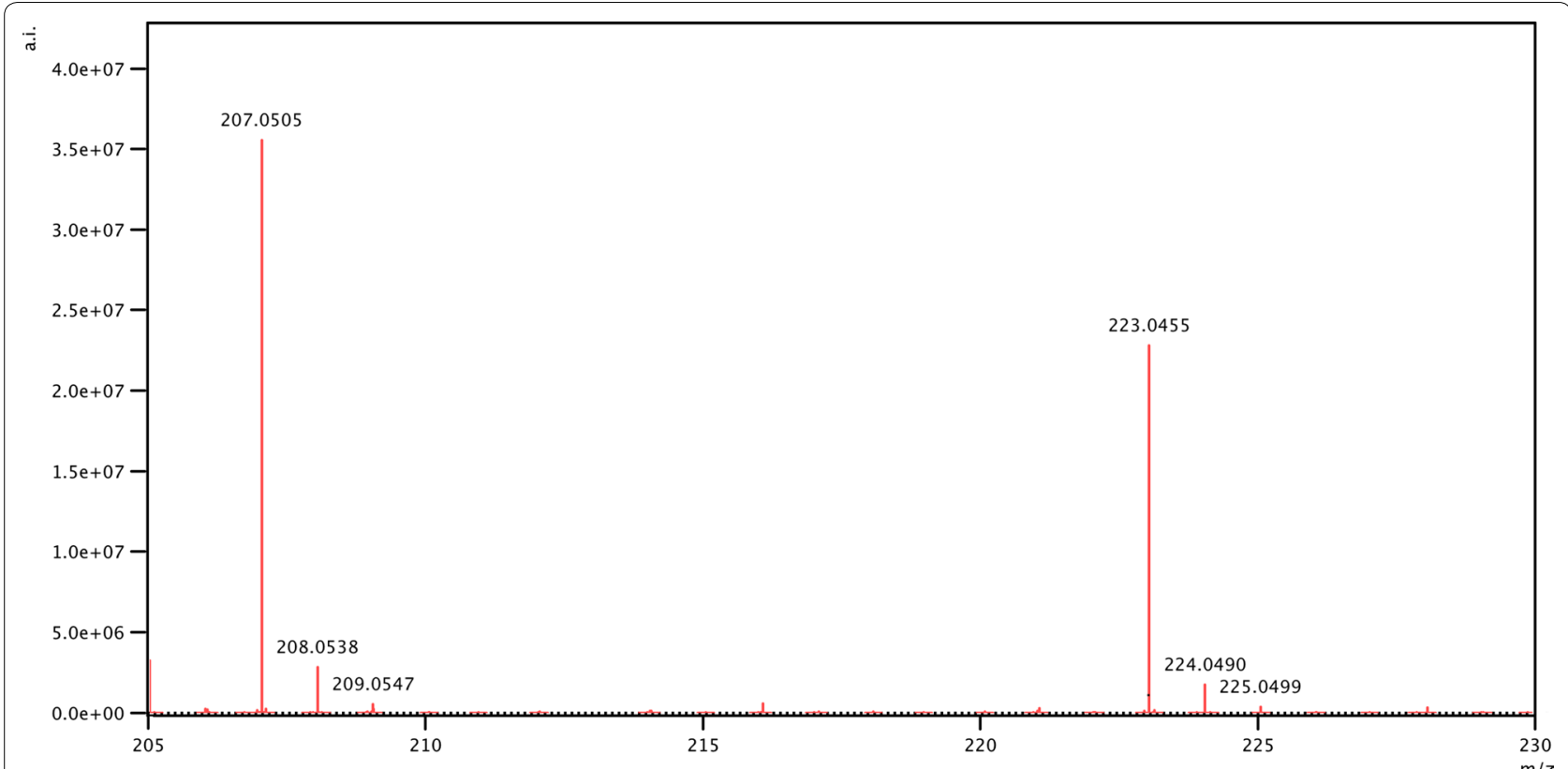

Fig. 5 LC-MS of glucuronoxylan after a one-pot sequential treatment with AxyAgu115 and GOOX-Y300A. Both MeGlcA (207.05 m/z) and methyl glucaric acid ( $223.05 \mathrm{~m} / \mathrm{z}$ ) were detected in the negative mode; the absolute intensity (a.i.) was shown

Other GOOX variants were produced in the previous work [20-22].

\section{Enzymatic hydrolysis and oxidation}

Glucuronoxylan (6\%) was incubated with AxyAgu115A $(10 \mu \mathrm{g} / \mathrm{mL}, 91 \mathrm{nM})$ and GOOX-Y300A $(10 \mu \mathrm{g} / \mathrm{mL}$, $179 \mathrm{nM}$ ) in $100 \mathrm{mM}$ Tris buffer $\mathrm{pH} 8.0$ at $40{ }^{\circ} \mathrm{C}$, rotated $15 \mathrm{rpm}$ (Hybaid mini hybridization oven MK II) for up to $72 \mathrm{~h}$. The reactions were then vacuum-filtered using 96-well filter plates $(0.22-\mu \mathrm{m}$ PVDF membrane) (Millipore, USA) in a Tecan liquid handler (500 mbar) (Tecan Trading AG, Switzerland). Enzymatic products in the flow-through were confirmed and quantified by mass spectrometry as well as by HPAEC-PAD and HPLC-RI/ UV analyses; whereas, different concentrations (up to $1 \%$, $\mathrm{w} / \mathrm{v}$ ) of the resulting xylan samples were incubated in MQ water with Novozymes xylanase NS51024 $\left(8 \times 10^{-4} \%\right.$, $\mathrm{w} / \mathrm{v}$ ) for $20 \mathrm{~min}$ at $40{ }^{\circ} \mathrm{C}$ at $700 \mathrm{rpm}$ in a thermomixer (Eppendorf, USA). The amount of reducing sugars was quantified by the PAHBAH method [31] while the release of xylose and xylo-oligosaccharides was quantified by HPAEC-PAD analysis after vacuum filtration.

The kinetics of GOOX-Y300A (16 nM) on MeGlcA and GlcA were measured using up to $50 \mathrm{mM}$ MeGlcA and GlcA in $0.3 \mathrm{M}$ Tris buffer $\mathrm{pH} 8.0$ at $40{ }^{\circ} \mathrm{C}$. The amount of methyl glucaric acid was determined by measuring the release of $\mathrm{H}_{2} \mathrm{O}_{2}$ using a previously published colorimetric assay [23].

\section{Quantification of MeGlcA from glucuronoxylan}

MeGlcA present in glucuronoxylan was released by a modified acidic methanolysis [32]. Briefly, glucuronoxylan $(10 \mathrm{mg})$, as well as MeGlcA $(1 \mathrm{mM})$, was treated with $1 \mathrm{~mL}$ of $2 \mathrm{M} \mathrm{HCl}$ in anhydrous methanol in glass vials at $100{ }^{\circ} \mathrm{C}$ for $3 \mathrm{~h}$. Samples were then dried by nitrogen flow, and re-dissolved in MilliQ water for HPAEC-PAD analysis.

\section{Docking analysis}

UCSF Chimera v 1.14 (https://www.cgl.ucsf.edu/chime $\mathrm{ra} /$ ) was used to energy-minimize the structures of MeGlcA (PubChem CID: 446874), glucose (PubChem CID: 5793) and GOOX wild-type (PDB ID: 2axr), as well as the structural model of GOOX-Y300A. The ligand and proteins were assigned AMBER ff14SB force field and AM1-BCC charges. Docking simulation was conducted using Autodock Vina v1.1.2 (http://vina.scripps.edu). Figures were generated by UCSF Chimera v 1.14.

\section{$\mathrm{H}_{2} \mathrm{O}_{2}$ inhibition assay}

AxyAgu115A $(10 \mu \mathrm{g} / \mathrm{mL})$ was incubated with $1 \%$ glucuronoxylan in $50 \mathrm{mM}$ Tris buffer $\mathrm{pH} 8.0$ in the presence of various $\mathrm{H}_{2} \mathrm{O}_{2}$ concentrations $(0.01-100 \mathrm{mM})$. MeGlcA (1 mM) was also incubated with the same $\mathrm{H}_{2} \mathrm{O}_{2}$ concentrations. The reactions were kept in the dark at $40^{\circ} \mathrm{C}$ at $500 \mathrm{rpm}$ for $16 \mathrm{~h}$ in a thermomixer (Eppendorf, USA), and then filtered using $30 \mathrm{kDa}$-cut-off filter centrifugal tubes. Catalase $(200 \mu \mathrm{g} / \mathrm{mL})$ 


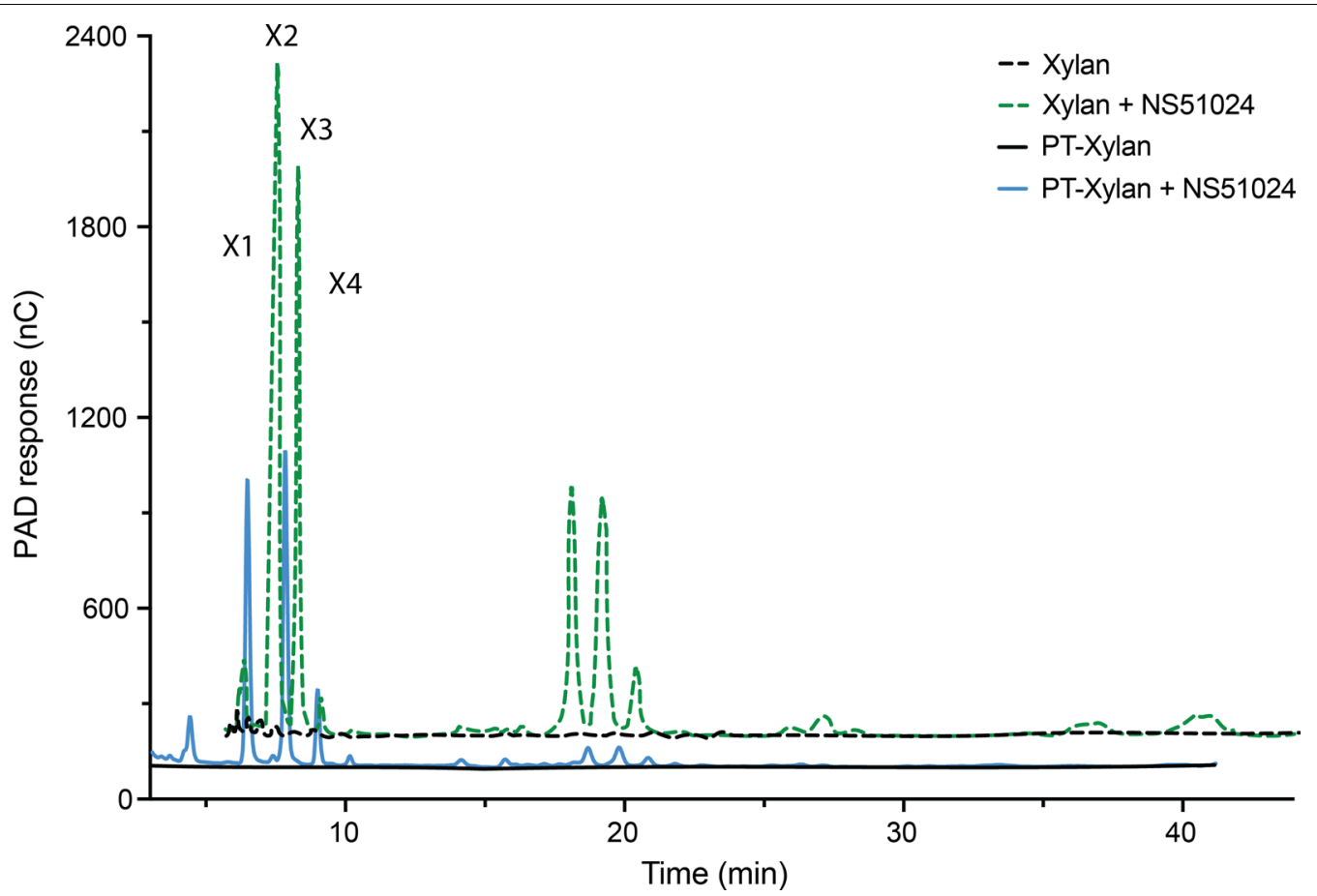

Fig. 6 HPAEC-PAD analysis for xylanase digestion of xylan (dash line), as well as AxyAgu115A and GOOX-Y300A-pretreated xylan (solid line). Xylan samples were treated using Novozymes NS51024; X1-X4 are xylose, xylobiose, xylotriose, and xylotetraose correspondingly. Peaks eluting after 15 min correspond to larger and/or charged oligosaccharides

was then added to the flow-through for 30-min incubation to remove $\mathrm{H}_{2} \mathrm{O}_{2}$ before HPAEC-PAD analysis.

\section{Anion-exchange chromatography}

Anion-exchange chromatography was performed using Dowex $1 \times 8$ anion-exchange resin (50-100 mesh) in a glass column $(2.6 \mathrm{~cm} \mathrm{ID} \times 30 \mathrm{~cm})$ connected to a BioLogic DuoFlow FPLC unit with a Quadtec UV detector (Bio-Rad, USA) with flow rates ranging from 1 to $3.0 \mathrm{~mL} / \mathrm{min}$. Milli-Q water was used as the primary eluent, and acidic sugars were eluted using a 0-2 $\mathrm{M}$ ammonium acetate $\mathrm{pH} 6.5$ gradient. Fractions containing eluted products were desalted and concentrated by lyophilization. The presence of sugar products in fractions was detected by spotting the sample on silica plates with aluminum backing (Sigma-Aldrich, USA), and visualized using the diphenylamineaniline stain [33].

\section{HPAEC-PAD and HPLC-RI/UV analyses}

Reaction samples were vacuum-filtered using $0.22-\mu \mathrm{m}$, PVDF filter plates (Millipore, USA) with a Tecan liquid handler (500 mbar) (Tecan Trading AG, Switzerland). The flow-through was collected to Nunc ${ }^{\mathrm{TM}}$ 96-well polypropylene microplates (Thermo Fisher Scientific, USA), and covered with Nunc ${ }^{\mathrm{TM}} 96$-well silicone cap mats. The presence of neutral and acidic sugars was detected using an ICS5000 HPAEC-PAD system (Dionex, USA) with a CarboPac PA1 $(2 \times 250 \mathrm{~mm})$ analytical column (Dionex, USA). The HPAEC-PAD samples were eluted at $0.25 \mathrm{~mL} /$ min using $\mathrm{NaOAc}$ gradient $(0-0.5 \mathrm{M})$ in $0.1 \mathrm{M} \mathrm{NaOH}$. The same filtered samples were also analyzed by a Dionex Ultimate 3000 HPLC (Dionex, USA) equipped with a UV detector (DAD-3000) and a Shodex RI-101 differential refractive index detector. The products were separated using a Bio-Rad HPX-87H column at $65{ }^{\circ} \mathrm{C}$, and eluted with $5 \mathrm{mM} \mathrm{H}_{2} \mathrm{SO}_{4}$ at $0.4 \mathrm{~mL} / \mathrm{min}$. Wavelengths were set at 210, 214, 260 and $280 \mathrm{~nm}$. Chromatograms were analyzed using Chromeleon 7.2 (Dionex, USA).

\section{Nanospray ionization ion-trap mass spectrometry (NSI-MS) and LC-MS analyses}

Reaction solutions were vacuum-filtered using $0.22-\mu \mathrm{m}$, PVDF filter plates (Millipore, USA), and then prepared in $50 \%$ methanol before being injected to a nano-ESI source on a Q-Exactive mass spectrometer (Thermo Scientific, USA) with a disposable pico-emitter. Samples were analyzed in a negative mode at a spray voltage of $2.5 \mathrm{kV}$, capillary temperature of $250{ }^{\circ} \mathrm{C}$, automatic gain control target of $1 \times 10^{6}$, injection time of $100 \mathrm{~ms}$, and resolution of 140,000. Spectra were analyzed using 
Qual Browser in Thermo Xcalibur (v2.2) software (Thermo Scientific, USA). For LC-MS analysis, the filtered samples were analyzed using a Q-Exactive mass spectrometer (Thermo Scientific, USA), equipped with an Ultimate 3000 HPLC system (Thermo Scientific, USA) and a Hypersil GOLD column $(50 \times 2.1 \mathrm{~mm})$ (Thermo Scientific, USA).

\section{Supplementary information}

Supplementary information accompanies this paper at https://doi. org/10.1186/s13068-020-01691-2.

Additional file 1. Additional figures.

\section{Abbreviations}

AxyAgu 115A: GH115 a-glucuronidase from Amphibacillus xylanus; GlcA: D-Glucuronic acid; GOOX: Gluco-oligosaccharide oxidase from Sarocladium strictum; MeGlcA: 4-O-Methyl D-glucuronic acid.

\section{Acknowledgements}

Not applicable.

\section{Authors' contributions}

ERM and TV conceived the study. TW conducted experiments and analyzed the data. TV and ERM wrote the manuscript. Both authors read and approved the final manuscript.

\section{Funding}

This work was funded by "SYNBIOMICS-Functional genomics and technoeconomic models for advanced biopolymer synthesis" with additional support from Ontario Genomics, Genome Quebec, and Genome BC (project \#10405, http://www.synbiomics.ca)".

\section{Availability of data and materials}

All data generated or analyzed during the study are included in this published article.

\section{Ethics approval and consent to participate}

Not applicable.

\section{Consent for publication}

Not applicable.

\section{Competing interests}

The authors declare that they have no competing interests.

\section{Author details}

1 Department of Chemical Engineering and Applied Chemistry, University of Toronto, Toronto, ON, Canada. ${ }^{2}$ Department of Bioproducts and Biosystems, Aalto University, Aalto, Kemistintie 1, 00076 Espoo, Finland.

Received: 8 January 2020 Accepted: 27 February 2020

Published online: 13 March 2020

\section{References}

1. Girio FM, Fonseca C, Carvalheiro F, Duarte LC, Marques S, BogelLukasik R. Hemicelluloses for fuel ethanol: a review. Bioresour Technol. 2010;101:4775-800

2. Pinto $P$, Evtuguin D, Neto C. Structure of hardwood glucuronoxylans: modifications and impact on pulp retention during wood kraft pulping. Carbohydr Polym. 2005;60:489-97.

3. Teleman A, Tenkanen M, Jacobs A, Dahlman O. Characterization of O-acetyl-(4-O-methylglucurono)xylan isolated from birch and beech. Carbohydr Res. 2002;337:373-7.
4. Werpy T, Petersen G. Top value added chemicals from biomass. Volume I-results of screening for potential candidates from sugars and synthesis gas. Oak Ridge: U.S. Department of Energy; 2004.

5. Dijkgraaf PJM, Verkuylen MECG, van der Wiele K. Complexation of calcium ions by complexes of glucaric acid and boric acid. Carbohydr Res. 1987;163:127-31

6. Kiely DE, Chen L, Lin TH. Hydroxylated nylons based on unprotected esterified D-glucaric acid by simple condensation reactions. J Am Chem Soc. 1994;116:571-8.

7. Rorrer NA, Dorgan JR, Vardon DR, Martinez CR, Yang Y, Beckham GT. Renewable unsaturated polyesters from muconic acid. ACS Sustain Chem Eng. 2016;4:6867-76.

8. Armstrong RD, Kariuki BM, Knight DW, Hutchings GJ. How to synthesise high purity, crystalline D-glucaric acid selectively. Eur J Org Chem. 2017;2017:6811-4.

9. Lee J, Saha B, Vlachos DG. Pt catalysts for efficient aerobic oxidation of glucose to glucaric acid in water. Green Chem. 2016;18:3815-22.

10. Solmi S, Morreale C, Ospitali F, Agnoli S, Cavani F. The oxidation of D-glucose to glucaric acid using Au/C catalysts. ChemCatChem. 2017:9:2797-806

11. Smith TN, Hash K, Davey CL, Mills H, Williams H, Kiely DE. Modifications in the nitric acid oxidation of D-glucose. Carbohydr Res. 2012;350:6-13.

12. Bozell JJ, Petersen GR. Technology development for the production of biobased products from biorefinery carbohydrates - the US Department of Energy's "Top 10" revisited. Green Chem. 2010;12:539-54.

13. Moon TS, Yoon SH, Lanza AM, Roy-Mayhew JD, Prather KL. Production of glucaric acid from a synthetic pathway in recombinant Escherichia coli. Appl Environ Microbiol. 2009;75:589-95.

14. Chen N, Wang JY, Zhao YY, Deng Y. Metabolic engineering of Saccharomyces cerevisiae for efficient production of glucaric acid at high titer. Microb Cell Fact. 2018:17:67.

15. Lee CC, Kibblewhite RE, Paavola CD, Orts WJ, Wagschal K. Production of glucaric acid from hemicellulose substrate by rosettasome enzyme assemblies. Mol Biotechnol. 2016;58:489-96.

16. Su HH, Guo ZW, Wu XL, Xu P, Li N, Zong MH, Lou WY. Efficient bioconversion of sucrose to high-value-added glucaric acid by in vitro metabolic gngineering. Chemsuschem. 2019;12:2278-85.

17. Petroll K, Care A, Bergquist PL, Sunna A. A novel framework for the cellfree enzymatic production of glucaric acid. Metab Eng. 2020;57:162-73.

18. Petroll K, Care A, Waterstraat M, Bergquist PL, Sunna A. Mixed-mode liquid chromatography for the rapid analysis of biocatalytic glucaric acid reaction pathways. Anal Chim Acta. 2019;1066:136-45

19. Yan R, Vuong TV, Wang W, Master ER. Action of a GH115 alpha-glucuronidase from Amphibacillus xylanus at alkaline condition promotes release of 4-O-methylglucopyranosyluronic acid from glucuronoxylan and arabinoglucuronoxylan. Enzyme Microb Technol. 2017;104:22-8.

20. Foumani M, Vuong TV, Master ER. Altered substrate specificity of the gluco-oligosaccharide oxidase from Acremonium strictum. Biotechnol Bioeng. 2011;108:2261-9.

21. Vuong TV, Master ER. Fusion of a xylan-binding module to gluco-oligosaccharide oxidase increases activity and promotes stable immobilization. PLoS ONE. 2014;9:e95170.

22. Vuong TV, Vesterinen AH, Foumani M, Juvonen M, Seppala J, Tenkanen M, Master ER. Xylo- and cello-oligosaccharide oxidation by gluco-oligosaccharide oxidase from Sarocladium strictum and variants with reduced substrate inhibition. Biotechnol Biofuels. 2013;6:148.

23. Lin S-F, Yang T-Y, Inukai T, Yamasaki M, Tsai Y-C. Purification and characterization of a novel glucooligosaccharide oxidase from Acremonium strictum T1. Biochim Biophys Acta. 1991;1118:41-7.

24. Bertaud F. Evaluation of acid methanolysis for analysis of wood hemicelluloses and pectins. Carbohydr Polym. 2002;48:319-24.

25. Li Y, Xue Y, Cao Z, Zhou T, Alnadari F. Characterization of a uronate dehydrogenase from Thermobispora bispora for production of glucaric acid from hemicellulose substrate. World J Microbiol Biotechnol. 2018;34:102.

26. Löwendahl L, Petersson G, Samuelson O. Formation of dicarboxylic acids from 4-O-methyl-D-glucuronic acid in alkaline solution in the presence and absence of oxygen. Carbohydr Res. 1975;43:355-9.

27. Vuong TV, Foumani M, MacCormick B, Kwan R, Master ER. Direct comparison of gluco-oligosaccharide oxidase variants and glucose oxidase: substrate range and $\mathrm{H}_{2} \mathrm{O}_{2}$ stability. Sci Rep. 2016;6:37356. 
28. Yazici EY, Deveci H. Factors affecting decomposition of hydrogen peroxide. In: Proceedings of the XIlth international mineral processing symposium. 2010. p. 609-16.

29. Hong CH, Kim SH, Kim YG, Shin NR. Method for producing glucaric acid. US9227904 B1; 2016.

30. Ryabova O, Vršanská M, Kaneko S, van Zyl WH, Biely P. A novel family of hemicellulolytic a-glucuronidase. FEBS Lett. 2009;583:1457-62.

31. Lever M. A new reaction for colorimetric determination of carbohydrates. Anal Biochem. 1972:47:273-9.

32. De Ruiter GA, Schols HA, Voragen AGJ, Rombouts FM. Carbohydrate analysis of water-soluble uronic acid-containing polysaccharides with high-performance anion-exchange chromatography using methanolysis combined with TFA hydrolysis is superior to four other methods. Anal Biochem. 1992;207:176-85.

33. MacCormick B, Vuong TV, Master ER. Chemo-enzymatic synthesis of clickable xylo-oligosaccharide monomers from hardwood 4-O-methylglucuronoxylan. Biomacromol. 2018;19:521-30.

\section{Publisher's Note}

Springer Nature remains neutral with regard to jurisdictional claims in published maps and institutional affiliations.
Ready to submit your research? Choose BMC and benefit from:

- fast, convenient online submission

- thorough peer review by experienced researchers in your field

- rapid publication on acceptance

- support for research data, including large and complex data types

- gold Open Access which fosters wider collaboration and increased citations

- maximum visibility for your research: over 100M website views per year

At BMC, research is always in progress.

Learn more biomedcentral.com/submissions 